\title{
El registro estadístico de las rupturas de unión en España. Evaluación e ideas para avanzar desde la demografía
}

\author{
Carles Simó* \\ Montse Solsona** \\ *Universitat de València. Estudi General. Departament de Sociologia i Antropologia social \\ carles.simo@uv.es \\ **Universitat Autònoma de Barcelona. Centre d'Estudis Demogràfics \\ msolsona@ced.uab.es
}

Recibido: 10-10-2007

Aceptado: 05-05-2008

\section{Resumen}

En España, la constitución de uniones y la conformación de unidades familiares ha sufrido un cambio espectacular en los últimos años, y sus características no son del todo perceptibles a los ojos del investigador social, entre otras razones, por no contar con fuentes de información adecuadas. En particular, el proceso de paulatina flexibilización de las uniones, que, con el fenómeno de la consensualidad, han perdido rigidez y han devenido más fáciles de constituir y de disolver, plantea el reto de confeccionar fuentes apropiadas de observación y cuantificación. Como consecuencia de ello, la observación del fenómeno de la ruptura de las uniones se convierte en un desafío todavía más difícil, si cabe. En este artículo, revisamos las fuentes estadísticas existentes en España para el estudio de las rupturas de las uniones, a fin de determinar hasta qué punto son representativas de la población afectada, mostrar sus carencias y proponer algunas ideas, inspiradas en la experiencia de otros países europeos, para avanzar en el registro estadístico de dicho fenómeno en España. Nuestro interés por mejorar la observación de las rupturas de unión en España surge de los interrogantes abiertos en estudios anteriores sobre el divorcio en España, sus factores determinantes y sus consecuencias, todo ello desde la perspectiva demográfica.

Palabras clave: ruptura de unión, divorcio, registro estadístico, fuentes demográficas.

Resum. El registre estadístic de les ruptures d'unió a Espanya. Avaluació $i$ idees per avançar des de la demografia

A Espanya, la formació de les unions i de les unitats familiars ha experimentat una transformació espectacular en els darrers anys. Les característiques de tots dos objectes d'estudi no són completament perceptibles per als investigadors, pel fet, entre altres raons, que no disposen de fonts adequades d'informació. Concretament, la progressiva flexibilització de les unions, que, amb el fenomen de la consensualitat, han perdut molta rigidesa i han esdevingut més faccils de constituir i de dissoldre, planteja el repte de confeccionar fonts d'observació i de quantificació apropiades. Consegüentment, l'observació de la ruptura de les unions esdevé un desafiament encara més difícil. En aquest article, s'hi revisen les fonts estadístiques que existeixen a Espanya per estudiar les ruptures d'unions, a fi de determi- 
nar fins a quin punt són representatives de la població afectada, mostrar-ne les mancances i proposar algunes idees inspirades en l'experiència d'altres països europeus per avançar en el registre estadístic d'aquest fenomen. El nostre interès perquè millori l'observació estadística de les ruptures d'unió a Espanya parteix de les preguntes que ens han sorgit en estudis anteriors sobre el divorci, sobre els factors determinants i les consequiències que comporta des de la perspectiva demogràfica.

Paraules clau: trencament d'unió, divorci, registre estadístic, fonts demogràfiques.

Abstract. The Statistical Record of Union Ruptures in Spain: Evaluation and Ideas for Improving from a Demographical Perspective

In Spain, the formation of unions and the configuration of family units has undergone a spectacular change over the last few years; moreover the characteristics of that change have not always been visible to the eyes of the social researcher, because, amongst other reasons, of the absence of adequate data sources. In particular, the thorough 'flexibilisation' of unions, which have become less permanent and much easier to constitute and dissolve, poses the problem of producing appropriate mechanisms of observation and measurement. As a consequence, studying the process of union rupture becomes ever more difficult. This article reviews existing statistical sources in Spain for the study of union rupture, in order to determine how far they cover the whole population affected and to highlight their deficiencies. It proposes some improvements for the statistical recording of this phenomenon in Spain, based on the experience of other European countries. Our interest in developing the study of union rupture in Spain, derives from questions raised in previous demographic studies of the determinants and consequences of divorce.

Key words: union rupture, divorce, statistical records, demographic sources.

\section{Sumario}

1. Introducción 4. Las rupturas de unión en España:

2. La Ley del divorcio de 1981 y su primera reforma de 2005

3. El Registro Civil y la protección de los derechos revisión de los instrumentos de observación existente

5. La experiencia europea en el registro estadístico del divorcio

Conclusiones e ideas para avanzar

Referencias bibliográficas

\section{Introducción}

En el momento de consultar las estadísticas publicadas por Eurostat sobre divorcios en Europa, el investigador atento advertirá la ausencia de datos adecuados para la medición de la incidencia del divorcio en España. Por ejemplo, en la edición de 2002 de la publicación European social statistics. Demography, España ofrece datos anuales para el período 1985-2000 del número total de divorcios y de la tasa bruta de divorcios, es decir, los que se dan por cada mil 
habitantes; pero el índice sintético de divorcio (en inglés Total divorce rate) y la duración media del matrimonio en el momento del divorcio ${ }^{1}$ sólo aparecen puntualmente para los años 1985, 1990 y 1995. Estas estimaciones puntuales se han obtenido a partir de las dos únicas encuestas demográficas retrospectivas disponibles en España, que incluyen información sobre historias de unión y biografías familiares, a saber: la Encuesta sociodemográfica de 1991 (ESD) y la Encuesta sobre fecundidad y familia de 1995 (FFS), y no a partir de las estadísticas del movimiento natural de la población, como sería en caso de seguir las recomendaciones de Naciones Unidas² (1998).

La precariedad estadística sobre el divorcio en España, teniendo en cuenta el contexto europeo, no se corresponde con un país que lo legalizó hace más de veinticinco años y que no se ha quedado atrás en las transformaciones familiares que otras zonas de Europa vivieron en décadas anteriores. En nuestro país, el descenso de la fecundidad y de la nupcialidad, así como el aumento de las parejas de hecho y del divorcio, se han producido de forma rápida y contundente, porque las generaciones nacidas a partir de 1955 marcaron rupturas en el comportamiento familiar que luego se generalizaron, de manera que hoy es posible hablar de cierta normalización de estos fenómenos (Treviño et al., 2000). A pesar de ello, en la medida en que su observación estadística es poco satisfactoria, los demógrafos que nos interesamos por el fenómeno de las rupturas de las uniones, a menudo nos sentimos incapacitados para hacer una contribución adecuada a la sociodemografía contemporánea de la familia comparada europea, para la cual la precisa datación de los acontecimientos demográficos es sumamente importante. Dicha datación permite, por una parte, calcular los indicadores adecuados de intensidad del fenómeno y, por otra parte, relacionar el acontecimiento del divorcio con otros factores que pueden ser entendidos, siempre desde la perspectiva del análisis sociodemográfico, como sus determinantes.

El análisis demográfico del fenómeno de las rupturas de unión se refiere tanto a la medición o cuantificación del fenómeno, como al conocimiento de sus características, de manera que pretende responder con precisión a las siguientes preguntas: ¿cuál es la probabilidad de que una unión se rompa en función de su duración?, ¿cómo varían las rupturas de unión en el tiempo?, ¿cuáles son los factores determinantes de las rupturas?, ¿cómo son las características más relevantes de las uniones que se rompen y los aspectos sociodemográficos de las personas que dejan de vivir en pareja? En España no es posible dar una respuesta precisa a estos interrogantes si antes no se mejoran los instrumentos de

1. El índice sintético de divorcio y la duración media del matrimonio son considerados como los indicadores demográficos más adecuados para medir la intensidad y el calendario del fenómeno.

2. Desde 1954, Naciones Unidas ha instado a la implantación de un registro civil en el que se inscriban todos los matrimonios y divorcios. El Registro Civil proporciona los datos de las estadísticas vitales, que, como se sabe, en España toman el nombre de movimiento natural de población. 
medición y de colección de información, con el objetivo de ir adaptándolos a las transformaciones que experimentan las uniones actualmente.

En el contexto actual de «flexibilización» de las uniones o, si se prefiere, de diversificación de las formas de unión mediante la aparición de nuevos modelos al lado del matrimonio convencional, recoger información sobre la constitución de uniones es una tarea compleja, puesto que estas uniones no son objeto de inscripción en el Registro Civil, sino en registros municipales paralelos, en caso de que la pareja acceda a registrar su unión consensual. Como consecuencia de ello, la observación estadística de las rupturas deviene todavía más compleja si cabe. Ello es consecuencia tanto de la falta de un registro completo de las rupturas matrimoniales, como de la dispersión de registros de la constitución de otras formas de unión.

Por nuestra parte, después de unos cuantos años dedicados a la investigación sobre el divorcio en España, nos parece que es el momento de hacer una evaluación, máxime ahora que se acaba de aprobar la primera reforma de la Ley del divorcio de 1981 que permite ya aligerar un proceso judicial de separación y divorcio que hasta hoy día ha sido extraordinariamente tedioso en España. No se trata de inventar nada nuevo, sino más bien de exponer el camino recorrido, detectar las carencias actuales e, inspirándonos en las iniciativas $\mathrm{y}$ en las soluciones adoptadas en países que nos son cercanos - tanto por el tipo de organización del estado, como por la similitud en las tendencias demográficas recientes- proponer ideas acerca del registro estadístico de las rupturas de las uniones en España, para situarnos lo más cerca posible de la observación continua, es decir, el registro continuo de los eventos. Asimismo, queremos argumentar la necesidad de diseñar nuevas fuentes que nos permitan estudiar los determinantes y las causas de las rupturas de unión.

En lo que respecta a la medición, este artículo da un repaso a los problemas de registro y observación del acontecimiento de las rupturas de uniones matrimoniales (con breves referencias a las uniones consensuales), así como a la información disponible en los recuentos de la población según su estado civil o su forma de convivencia, es decir, el recuento de las personas separadas y divorciadas. Paralelamente, se considera la recogida de información para el estudio de las causas de las rupturas, así como sus efectos más inmediatos; siempre desde una perspectiva meramente demográfica.

\section{La Ley del divorcio de 1981 y su primera reforma de 2005}

El 29 de junio de 2005, el Pleno del Congreso de los Diputados aprobó la primera reforma ${ }^{3}$ de la Ley del divorcio de 1981, que, entre otras cosas, da luz verde al divorcio sin necesidad de separación previa. Los antecedentes de la Ley de 1981, que legalizó, en la última etapa democrática en España, el divor-

3. La ley que entró en vigor el día 9 de julio de 2005 es la Ley 15/2005, por la que se modifica el Código Civil en materia de separación y divorcio, y la Ley de enjuiciamiento civil (que se refiere al procedimiento). 
cio y la separación matrimonial, se encuentran en la Constitución de la Segunda República de 9 de diciembre de 1931, que introdujo el divorcio propiamente dicho en su articulo 43, y la Ley de 2 de marzo de 1932, que establecía un sistema de divorcio sanción, pero también admitía el divorcio por mutuo acuerdo, lo cual supuso una novedad en el derecho comparado de la época. Posteriormente, durante la dictadura franquista, la Ley de 12 de marzo de 1938 derogó la de 1932 - y anuló todos los divorcios concedidos durante el período republicano-, con lo cual dejó vigentes los preceptos anteriores del Código Civil, de manera que, durante cuatro décadas, volvió a imponerse la indisolubilidad del matrimonio como principio de «orden público» en el seno del ordenamiento jurídico español.

Con la transición democrática, la Constitución de 1978 estableció que la Ley debía regular las formas de matrimonio, la edad de los contrayentes, la capacidad para contraerlo, los derechos y deberes de los cónyuges, las causas de separación y disolución y sus efectos. La Ley 30/1981, de 7 de julio, que contempla el matrimonio tanto desde el punto de vista de su celebración como de su disolución en las tres formas de nulidad, separación y divorcio, modificó la regulación del matrimonio en el Código Civil y determinó el procedimiento a seguir en las causas de nulidad, separación y divorcio.

En España, durante casi un cuarto de siglo, el proceso legal de separación y divorcio ha sido largo y multietápico (separación primero, divorcio después). El divorcio se concebía como el último recurso al que podían acogerse los cónyuges y sólo cuando era evidente que, tras un dilatado período de separación, su reconciliación ya no era factible. Se exigía la demostración del cese efectivo de la convivencia conyugal o de la violación reiterada o grave de los deberes conyugales, una suerte de pulso impropio de los tiempos en que vivimos que la ley imponía a los esposos, obligados bien a perseverar públicamente en su desunión, bien a renunciar a tal iniciativa, lo cual llegó incluso a forzar reconciliaciones de más que dudoso éxito.

Sin embargo, la Ley 15/2005, de 9 de julio, establece eliminar el requisito de separación como paso previo a la obtención del divorcio y acelerar su proceso, de forma que, en caso de mutuo acuerdo por parte de los cónyuges, el divorcio pueda hacerse efectivo en dos o tres meses. Otra modificación interesante de esta reforma de la Ley del divorcio es la eliminación de las secuelas del modelo anterior de separación sanción, es decir, del requisito de culpabilidad, de manera que un cónyuge podrá iniciar de forma unilateral un proceso de divorcio. Según dice literalmente la nueva ley: «La reforma que se acomete pretende que la libertad, como valor superior de nuestro ordenamiento jurídico, tenga su más adecuado reflejo en el matrimonio. El reconocimiento por la Constitución de esta institución jurídica posee una innegable trascendencia, en tanto que contribuye al orden político y la paz social, y es cauce a través del cual los ciudadanos pueden desarrollar su personalidad».

Así, la nueva ley persigue ampliar el ámbito de libertad de los cónyuges en lo relativo al ejercicio de la facultad de solicitar la disolución de la relación matrimonial. De la misma manera, de acuerdo con los valores y los principios cons- 
titucionales que configuran el matrimonio como un derecho, la nueva ley reconoce el cambio evidente en el modo de concebir las relaciones de pareja en nuestra sociedad, al hacer que el derecho a no continuar casado no dependa de la concurrencia de causa alguna, sino de la voluntad expresada en este sentido. De manera que la reforma establece eliminar el requisito de separación como paso previo a la obtención del divorcio y acelerar el proceso, de forma que, en caso de mutuo acuerdo por parte de los cónyuges, el divorcio pueda hacerse efectivo en dos o tres meses. El divorcio podrá solicitarse tres meses después de la boda (hasta ahora había que esperar un año) sin necesidad de haberse separado, lo cual reducirá considerablemente costes de todo tipo: procesales, de inversión en tiempo, económicos, emocionales, etc. La eliminación del requisito de culpabilidad, lo que permite que un cónyuge pueda iniciar de forma unilateral un proceso de divorcio, es una innovación a tener en cuenta.

Esta reforma también regula la custodia compartida de los hijos (hasta ahora sin regular), de manera que los niños podrán vivir alternativamente con el padre y la madre. Se creará un fondo que garantice las pensiones a los hijos y se regulará la posibilidad de acudir a la mediación familiar voluntaria (habrá que hacer leyes específicas en cada caso). Esta reforma de la Ley del divorcio, publicada en el Boletín Oficial del Estado como la Ley 15/2005, de 9 de julio, entró en vigor de forma inmediata para beneficiar no solamente a los procesos iniciados a partir de esta fecha, sino a todos aquéllos que estuvieran pendientes de resolución.

Por lo que a la recolección y disponibilidad de datos se refiere, la elaboración de estadísticas de divorcio, a partir de la aprobación de la Ley de 1981 y de su implementación en el mes de septiembre de 1981, ha constituido un largo camino, pues los esfuerzos por armonizar la actividad de los legisladores, los administradores de justicia y los responsables de elaborar las estadísticas judiciales y demográficas ha llevado demasiado tiempo. En efecto, hubo que esperar hasta el año 1996 para llegar a disponer de algo más que de la escueta información acerca del número y del tipo de sentencias — según grado de acuerdo entre cónyuges - de los procesos de separación, divorcio y nulidad, publicada en la memoria anual del Consejo General del Poder Judicial. Finalmente, en 1996, en virtud del acuerdo suscrito entre el Instituto Nacional de Estadística de España (INE) y el Consejo General del Poder Judicial (CGPJ), el 14 de febrero de 1995, el INE, en su publicación Estadísticas judiciales, incluye por primera vez datos sobre los procesos y las sentencias de ruptura de las uniones. Estos datos se recogen mediante el Boletín estadístico ${ }^{4}$ que fue diseñado ad hoc y reúnen información sobre los procesos, las sentencias, las características de las uniones y las características de los cónyuges. Estos boletines son cumplimentados por los juzgados y remitidos al CGPJ, el cual es responsable de entregarlo al INE.

4. La información contenida en el Boletín estadístico de sentencias de separaciones, divorcios y anulaciones ha cambiado ligeramente desde su implementación hasta la actualidad (véase la tabla 1). 
La cobertura de los boletines estadísticos de sentencias de separaciones, divorcios y anulaciones en el año 2001, según estimaciones hechas por el propio INE, alcanza un $73,7 \%$ de las sentencias dictadas. Pero con la reciente reforma de la Ley del divorcio y la agilización de los procesos judiciales, confiamos en que vaya mejorando el registro estadístico de las sentencias de separación y divorcio, en la medida en que en los juzgados, que tienen la responsabilidad de cumplimentar el boletín correspondiente, el ritmo de trabajo sea más llevadero. De ser así, las condiciones para desarrollar una buena demografía del divorcio también mejorarán sustancialmente.

\section{El Registro Civil y la protección de los derechos}

\subsection{Las recomendaciones de Naciones Unidas}

Naciones Unidas viene instando, desde 1954, a la implantación de un registro civil en el que se inscriban todos los matrimonios y divorcios. Con ello se busca que las actas de matrimonio y divorcio se usen administrativamente como prueba tangible del reconocimiento oficial del proceso de formación de la familia, al mismo tiempo que constituyan fuentes de información privilegiadas para la comprensión de la evolución de la nupcialidad y la divorcialidad a lo largo del tiempo.

Con esta intención, Naciones Unidas define el sistema de estadísticas vitales como el proceso total consistente en:

1) Reunir información sobre la frecuencia de acaecimiento de determinados sucesos vitales definidos, así como de las características pertinentes de los propios sucesos y de la o las personas de que se trata.

2) Compilar, analizar, evaluar, presentar y difundir esos datos en forma estadística (Naciones Unidas, 1998). Por otra parte, los sucesos vitales que Naciones Unidas recomienda integrar en el Registro Civil son los siguientes: nacimientos, adopciones, legitimaciones, reconocimientos; defunciones y defunciones fetales; matrimonios, divorcios, separaciones y anulaciones.

En lo que aquí nos concierne, cabe señalar que las actas de matrimonio y divorcio constituyen la documentación en la que se funda el estado civil de las personas - el mismo que confiere la posibilidad de casarse a los solteros, los divorciados y los viudos. De este modo, por un lado, las actas de divorcio o separación son importantes para establecer el derecho de una persona a contraer un nuevo matrimonio, así como para quedar liberada de las obligaciones financieras y de otra índole que pueda contraer la otra parte. Y, por otro lado, la certificación del estado civil de las personas deviene imprescindible para fines tales como la percepción de pensiones alimenticias, la solicitud de beneficios tributarios, el suministro y la asignación de vivienda, y otras prestaciones relacionadas con el estado matrimonial de una pareja, así como el cambio de nacionalidad por causa de matrimonio. 
Por lo general, las preocupaciones de la sociedad se superponen con los usos que hacen las personas de las actas del Registro Civil. Pero dichas preocupaciones también se relacionan con los conceptos más generales del desarrollo humano, los derechos humanos y la protección del niño, de la mujer y la familia. De ahí el valor protector que se confiere a los registros de nacimientos vivos, matrimonios y divorcios, y que ha sido refrendado oficialmente por las Naciones Unidas en varias medidas 5 . En resumen, podemos afirmar que, en la sociedad, existe un interés general en la universalización de la cumplimentación de las actas del Registro Civil y en la utilización de las mismas como documentos garantes de derechos. Todo ello también afecta, y de manera especial, al registro del fenómeno que aquí nos interesa: las rupturas matrimoniales.

Finalmente, y por lo que se refiere a las fuentes estadísticas para el estudio de las rupturas de unión, Naciones Unidas, en sus recomendaciones, alerta de la dificultad de utilizar fuentes estadísticas diferentes del Registro Civil, como censos y encuestas, ya que los datos contenidos en éstos últimos nunca alcanzan la totalidad del universo con la misma garantía que consigue el Registro Civil mediante la universalización de la cumplimentación y mediante la naturaleza de documento garante de derechos. Veamos ahora cuál es la situación del registro de las rupturas de unión en España.

\subsection{Registro civil y movimiento natural de la población en España}

En nuestro país, las estadísticas del movimiento natural de la población, que se refieren básicamente a los nacimientos, los matrimonios y las defunciones ocurridos en el territorio español o de españoles en el extranjero, constituyen uno de los trabajos de más tradición del INE. El primer volumen se publicó en 1863 por la Junta General de Estadística del Reino y contenía datos relativos al período que iba de 1858 a 1861, obtenidos a partir de los registros parroquiales. Desde entonces se viene publicando en España información de los fenómenos demográficos sin más interrupción que durante los años $1871 \mathrm{a}$ 1885, época de implantación del Registro Civil, de donde se obtendrían en lo sucesivo los datos para estas estadísticas.

Puesto que la ruptura matrimonial (junto con la defunción de al menos uno de los cónyuges) supone el fin del matrimonio, a un observador externo podría parecerle que la forma más lógica de recabar información sobre el fenómeno de las rupturas matrimoniales sería la misma que para los matrimonios — en cuyo registro se anota su disolución ${ }^{6}$ —, es decir, vía registro civil. Así,

5. Declaración Universal de Derechos Humanos (1948), Declaración de los Derechos del Niño (1959) y Pacto Internacional de Derechos Civiles y Políticos (1966).

6. En los libros físicos del Registro Civil, se hace una anotación al margen del acta matrimonial con la fecha de la sentencia de separación o de divorcio. En cuanto la informatización del Registro Civil sea un hecho en toda España, será posible, con los años, disponer de la información necesaria para calcular el índice sintético de divorcialidad. 
Tabla 1. Información de los boletines estadísticos de sentencias de anulaciones, separaciones y divorcios

\begin{tabular}{|c|c|c|c|c|}
\hline & $1995-1998$ & $1999-2003$ & 2004 & 2005 \\
\hline \multicolumn{5}{|l|}{ Sobre el proceso judicial } \\
\hline $\begin{array}{l}\text { Identificación juzgado, número } \\
\text { y provincia }\end{array}$ & & & & (provincia) \\
\hline \multicolumn{5}{|l|}{$\begin{array}{l}\text { Trimestre y año de recogida } \\
\text { de la información }\end{array}$} \\
\hline \multicolumn{5}{|l|}{ No de expedient } \\
\hline \multicolumn{5}{|l|}{ No de sentencia } \\
\hline \multicolumn{5}{|l|}{$\begin{array}{l}\text { Fecha de presentación de la demanda } \\
\text { (día, mes y año) }\end{array}$} \\
\hline Fecha de la sentencia (día, mes y año) & & & & \\
\hline
\end{tabular}

Datos del matrimonio

Fecha de la celebración (día, mes y año)

Lugar de celebración (provincia y municipio)

Número de hijos vivos menores de edad

Número de hijos menores de edad

Total de hijos nacidos vivos, incluidos los menores

Datos del esposo y de la esposa

Fecha de la celebración (día, mes y año)

Estado civil al contraer el actual matrimonio (soltero/a, viudo/a, divorciado/a)

DNI, NIE o pasaporte

Información sobre la sentencia

Causa reconocida en la sentencia: nulidad (6 categorías), separación (9) o divorcio (6)

Existencia de separación civil legal previa en caso de divorcio

Apelación de la sentencia

Tipo de fallo de la sentencia (separación mutuo acuerdo/contenciosa; divorcio consensuado/no consensuado, nulidad matrimonial)

\section{Información sobre el demandante}

Esposo, esposa o ambos

Información sobre pensiones

Pensión compensatoria con cargo a (esposo o esposa)

Pensión alimenticia con cargo a (padre, madre o ambos)

Información disponible en los ficheros de microdatos desde 1996.

Fuente: Boletín Estadístico, Modelo E. D.; INE. Servicio de Estadísticas Judiciales. Consejo General del Poder Judicial. 
el Registro Civil, igual que remite al INE información de los matrimonios registrados, podría informar de las rupturas de las uniones matrimoniales acaecidas. Sin embargo, como en otros países de nuestro entorno, el divorcio y la separación son eminentemente procesos judiciales y, por lo tanto, son competencia del poder judicial. De manera que la cumplimentación del Boletín estadístico de sentencias de separación, divorcio y nulidad (tabla 1) —análogo al Boletin de parto, matrimonio y defunción que el Registro Civil remite al INEes una responsabilidad de los juzgados, y no del Registro Civil, cada vez que se dicta una sentencia que legaliza la ruptura de una unión matrimonial. En virtud del ya mencionado acuerdo entre el INE y el CGPJ firmado en 1995, la elaboración de datos estadísticos de divorcios y separaciones, mediante la implementación de los boletines mencionados, queda en manos de la administración de justicia, si bien el Instituto Nacional de Estadística se encarga de recopilarlos, tabularlos y publicitarlos, como hace con las estadísticas del Registro Civil de nacimientos, defunciones y matrimonios.

Ciertamente, en España, las estadísticas del movimiento natural de la población (MNP) recogen abundante información del Registro Civil sobre los nacimientos, las defunciones y las defunciones fetales y los matrimonios ${ }^{7}$. Sin embargo, el resto de acontecimientos vitales que el reconocimiento de derechos al que van asociados son objeto de debate social (divorcios, separaciones $\mathrm{y}$ anulaciones, adopciones, legitimaciones, reconocimientos), no se incluyen como tales en el MNP8.

En conclusión, la inclusión del divorcio en el Registro Civil no está resuelta en España, al menos en los términos recomendados por Naciones Unidas, pues no cumple con el objetivo de constituir la fuente estadística para medir la amplitud del fenómeno y conocer sus características.

\section{Las rupturas de union en España: revisión de los instrumentos de observación existentes}

En este apartado, nuestra intención es revisar los problemas de observación de las rupturas de uniones a partir de las fuentes estadísticas existentes, teniendo en cuenta las dificultades relativas a la observación del momento de ruptura y los problemas de observación según el tipo de unión. Analizaremos también las carencias y las virtudes de las distintas fuentes estadísticas que recogen información sobre el evento (la ruptura de la unión), como también sobre la población separada y divorciada. En último lugar, abordaremos los problemas de

7. En un futuro próximo, en virtud de la reciente Ley de julio de 2005 que legaliza los matrimonios entre personas del mismo sexo en España, el Registro Civil, y consiguientemente el MNP, deberá dar cuenta también de los matrimonios homosexuales.

8. Por ejemplo, las adopciones realizadas por una mujer sola, hasta primeros de julio de 2005 , momento en el que se aprueba una importante reforma del Código Civil, debían ser inscritas con dos progenitores: la madre real y un padre inventado. Afortunadamente, esta reciente reforma ha puesto fin a una práctica absolutamente anacrónica. 
observación de los determinantes y de las consecuencias de las rupturas de unión a partir de encuestas.

\subsection{El registro del acontecimiento en la estadísticas judiciales}

Actualmente, los demógrafos encuentran dificultades serias a la hora de responder preguntas de gran importancia como: «¿Cuántas uniones se rompen anualmente en la sociedad española?», "¿En qué momento preciso se rompen?».

En España, la inscripción del divorcio en el Registro Civil queda reducida a una nota marginal en el acta matrimonial. Esta nota marginal no contiene ninguna información, aparte de la fecha y el tipo de ruptura, con lo que no puede ser objeto de ningún tipo de tratamiento estadístico conducente a averiguar el perfil sociodemográfico de los protagonistas o las circunstancias de la ruptura. En cambio, sí se encuentra cierta información en el Boletín estadistico de las sentencias de divorcio, separación y nulidad que el CGPJ debe remitir al INE. En él, se recogen datos relativos al proceso (duración, demandante, tipo de sentencia y existencia de separación civil previa), al matrimonio (básicamente fecha y lugar de celebración e hijos habidos en él), escasos datos sociodemográficos de la esposa y del esposo (estado civil anterior al matrimonio que se disuelve y edad en el momento de casarse) y, por último, datos sobre el tipo de pensiones acordadas en la sentencia. En la tabla 1 adjunta, damos cuenta de las modificaciones en el contenido del boletín.

El estudio de las características sociodemográficas de las uniones y de las personas que dejan de vivir en pareja es posible gracias a la labor realizada por el INE de cotejo con las propias estadísticas publicadas por el CGPJ. De hecho, la cobertura de los boletines que ha recogido el INE - y que ha puesto al alcance de los investigadores en ficheros de microdatos- desde 1996 se acerca al $70 \%$ de las sentencias dictadas por el CGPJ.

Las estadísticas que difunde el INE en base a los datos del CGPJ vuelcan el número de sentencias judiciales que conllevan las rupturas de uniones matrimoniales, lo que se acerca bastante a la cantidad de eventos de rupturas; sin embargo, quedan al margen del recuento todas las rupturas matrimoniales que no generan un proceso judicial (por ejemplo, aquéllas totalmente consensuadas por ambos cónyuges). Cabe apuntar además que, aparte de las rupturas matrimoniales, las rupturas de otros tipos de unión, como las parejas de hecho, únicamente pueden generar procesos judiciales en caso de contencioso sobre bienes comunes y custodia de hijos; y si se da este caso, dichos procesos tampoco forman parte de las estadísticas judiciales de divorcios y separaciones legales, porque no lo son, sino que aparecen dentro de la categoría genérica de "procesos judiciales de familia». $\mathrm{O}$ sea, que por esta vía tampoco se pueden rescatar «estadísticamente» estas rupturas.

\subsection{La observación del momento de la ruptura}

Las rupturas que aparecen en las estadísticas judiciales, como ya se ha avanzado, son solamente una parte - aunque representen la mayoría - de las rup- 
turas de uniones matrimoniales. Son aquéllas en las que aparecen aspectos conflictivos o en las que existe la voluntad de contraer un nuevo matrimonio por parte de uno o de los dos miembros de la pareja. Pero, ¿a qué momento de la ruptura se refieren estas estadísticas? La respuesta aquí es obvia: se refieren a sentencias de procesos judiciales iniciados después del rompimiento de facto de la unión y, por lo tanto, son dictadas en una fecha muy lejana al acontecimiento de la ruptura.

En este estado de cosas, para los demógrafos, resulta problemático no solamente calcular la intensidad del fenómeno, dada la dificultad de llegar a la totalidad de los eventos, sino también establecer el momento preciso en que la separación de los cónyuges se hizo efectiva a través de la información estadística existente.

Al preguntar a los protagonistas de una ruptura de una unión matrimonial, podíamos recabar diferentes fechas en relación con el proceso de divorcio: 1) el momento en el que cesó la convivencia de los cónyuges, pero el tema se complica en el caso de los que nunca han compartido el mismo techo de forma permanente 9 y en el de las parejas que se han separado y han reiniciado su convivencia en varias ocasiones; 2) el día en que se inició un proceso legal de separación; 3) el día en que se inició el proceso legal de divorcio; 4) el día en que se dictó la sentencia de separación, y 5) el día de la sentencia de divorcio. Como hemos visto, los tiempos (los momentos) pueden ser muchos y el conocimiento que podamos tener de este fenómeno dependerá, por tanto, de las estadísticas de las que se disponga.

En los estudios demográficos de la separación y del divorcio, nos interesa privilegiar aquel momento en el que se toma la decisión de acabar con la convivencia. Pero éste no queda registrado en ninguna parte y únicamente puede conocerse mediante la elaboración de una encuesta.

En las fuentes existentes, los momentos por los que atraviesa la ruptura y que resultan objeto de registro son tres: la presentación de la demanda, la admisión de la misma y la sentencia judicial, si bien las estadísticas judiciales únicamente dan cuenta de las fechas de admisión de la demanda y de la sentencia. Insistimos en que éste es un tema de especial relevancia en la demografía contemporánea, ya que es difícil avanzar en el estudio de los fenómenos cuando no se cuenta con una datación precisa de los acontecimientos.

Este problema se agrava cuando valoramos el tiempo que puede llegar a discurrir entre el momento de la presentación de la demanda y el del dictamen de la sentencia, máxime cuando sabemos que, en un altísimo porcentaje (entre un $70 \%$ y un $80 \%$ en el período 1996-2002), al proceso de divorcio le precede un proceso de separación (Solsona y Simó, 2007).

Sin embargo, otras fuentes alternativas, a saber, la Encuesta sociodemográfica (ESD) de 1991 y la Encuesta de fecundidad y familia (FFS) de 1995, han brindado una información útil sobre el momento de la ruptura: aquél en

9. Es el caso de lo que, en terminología inglesa, se conoce como Living Apart Together. 
el que acabó la convivencia. Pero, con posterioridad a esta fecha, no existe ninguna fuente de información que permita calcular los índices más adecuados para medir la intensidad y el calendario del fenómeno de las rupturas de uniones en España: el índice sintético y la duración media ${ }^{10}$.

\subsection{El registro de la ruptura según el tipo de unión}

Si es difícil presentar la historia de las rupturas matrimoniales en España desde la legalización del divorcio, es fácil de imaginar que el estudio de las rupturas de las uniones consensuales - de las cuales apenas conocemos su importancia numérica - deviene una realidad todavía más escurridiza. De hecho, para el conjunto de España, el primer recuento oficial de este tipo de uniones lo facilitó el INE en verano de 2004, a partir de una explotación del último censo de población de $2001^{11}$. Con anterioridad a esa fecha, únicamente se contaba con estimaciones puntuales de algunas encuestas nacionales (ESD y FFS) o de carácter local (como la Encuesta metropolitana de Barcelona).

Cierto es que, desde hace algunos años, existen registros municipales de parejas de hecho en algunos municipios. En el caso de Cataluña, por ejemplo, estos registros se rigen por la Ley de uniones estables de pareja de 1998, regulación completa de las parejas de hecho, con independencia de su orientación sexual, con matizaciones específicas para cada tipo de unión: heterosexual y homosexual ${ }^{12}$. La Ley catalana de parejas de hecho regula básicamente las competencias del derecho civil que corresponden a la Generalitat de Cataluña, quedando excluidas las que no son competencias autonómicas (cuestiones propias del derecho penal, de carácter laboral y las relativas a la seguridad social).

De ello se desprende que las posibilidades de observación estadística de los procesos de ruptura difieren enormemente según el tipo de unión: matrimonio, pareja estable con convivencia, pareja estable sin convivencia, etc., sin nombrar los procesos paralelos que rigen la ruptura canónica de parejas que se casaron por la Iglesia católica. De hecho, en el caso de las uniones consensuales, la eventual ruptura únicamente es observable si se recurre al juzgado para decidir la custodia de hijos comunes o para resolver conflictos en la separación de bienes comunes. Pero como quiera que no existe un boletín estadístico análogo a las sentencias de separación y divorcio, en la actualidad, no es posible estudiar las circunstancias de las rupturas de las parejas de hecho tal como podemos hacerlo con las separaciones y los divorcios legales. Es de desear que las estadísticas judiciales mejoren progresivamente y que, igual que la activi-

10. El Panel de hogares de la Unión Europeo, cuya última oleada disponible es la del 2001, no permite el estudio de la ruptura de uniones, ya que ofrece un número de casos demasiado pequeño.

11. Este primer recuento publicado en octubre de 2004 arroja una cifra igual a 563.000 parejas de hecho, de las cuales $10.474(0,11 \%)$ son uniones homosexuales.

12. Esta ley se ve complementada por la que entró en vigor en julio de 2005: la ley que permite las uniones matrimoniales homosexuales, que serán inscritas en el Registro Civil. 
dad legislativa se ha adaptado a las nuevas realidades sociales, se vayan desarrollando instrumentos de observación que permitan conocer estos procesos de una forma más completa.

\subsection{El recuento de la población según el estado de separado o divorciado: los censos y las encuestas}

Los censos y las encuestas permiten conocer cuantitativamente las personas cuyo estado era separado o divorciado en un momento dado cuando se pretende levantar un censo, lo cual es equivalente a hacer una fotografía un día determinado de todos los divorciados y divorciadas que habitan en cada distrito censal.

Las fuentes que hemos utilizado en estudios anteriores son: el Censo de población de 1991, la Encuesta sociodemográfica de 1991 y la Encuesta española de fecundidad y familia de 1995. De los censos de población de 1991 y de 2001, es posible utilizar el fichero de microdatos que contiene una muestra del mismo del 10\% para analizar el perfil sociodemográfico de la población divorciada y separada. Pueden cuantificarse así los efectivos y comparar las características sociodemográficas de la población separada y divorciada en relación con la población casada, controlando la edad y el sexo. Como variables explicativas, se pueden elegir el nivel de instrucción, la relación con la actividad, la profesión, la situación profesional y el tamaño del municipio. De esta manera, se puede averiguar si la población separada y divorciada presentaba un perfil específico, en relación con la casada, que pudiera denotar un cierto proceso de selección social de la población susceptible de padecer un divorcio o una separación.

La Encuesta sociodemográfica de 1991, que se llevó a cabo al mismo tiempo que el Censo de 1991, constituye una muestra representativa de la población española de aquel año. La ESD es una fuente privilegiada para el estudio de los eventos demográficos desde una perspectiva biográfica, ya que contiene información retrospectiva sobre la biografía familiar, educativa, laboral y migratoria de cerca de 156.000 individuos de diez y más años de edad y, además, es representativa para cada comunidad autónoma ${ }^{13}$. Su tamaño es una ventaja frente a otras encuestas europeas (FFS, PHOGE y ESS) ${ }^{14}$.

La Encuesta española de fecundidad y familia (FFS, del inglés Fertility and Family Survey), de 1995, se basó en una muestra de hogares representativa a

13. En estudios anteriores, basados en datos de la encuesta sociodemográfica de 1991, hemos trabajado con un fichero seleccionando las primeras uniones del conjunto del Estado, ya fueran matrimoniales o de cohabitantes, que eran cerca de 108.000 y entre las cuales se registraba un total de 4.020 rupturas (ya fueran divorcios, separaciones legales o de hecho) (Houle et al., 1999; Treviño et al., 2000).

14. La muestra del Panel de hogares de la Unión Europea (PHOGE), que viene realizándose en España desde 1994, fue de 12.317 personas en el año 2000. La primera ola de la Encuesta social europea (ESS) se realizó en el año 2002 y en el caso de España se entrevistaron a 1.715 personas. 
nivel nacional, con un total de 4.021 mujeres y 1.991 hombres de edades entre 18 y 49 años (Delgado y Castro Martín, 1999). La FFS ofrece datos por generaciones muy interesantes sobre el acaecimiento de distintos tipos de unión (matrimonio y uniones consensuales), así como de las rupturas a las distintas edades que, además, pueden ser interpretadas en un marco comparativo con todos los países que participaron en la FFS ${ }^{15}$.

\subsection{Factores determinantes. Las encuestas como fuentes idóneas para el estudio de las causas de las rupturas de uniones}

Hablar de las causas del divorcio o, para ser algo más precisos con la terminología, de los factores sociodemográficos determinantes del divorcio, es algo muy diferente de las causas legales y de lo que comúnmente se entiende como «el motivo de una separación». En la legislación española vigente, hasta la aprobación de la reciente reforma (9 de julio de 2005) de la Ley del divorcio de 1981, se contemplaban distintas causas legales de nulidad, separación y divor$\mathrm{cio}^{16}$, si bien, en la práctica, los jueces tendían a considerar o a aceptar la falta de amor como causa suficiente de divorcio.

En demografía, el estudio de las causas es mucho más reduccionista, pues se trata de mostrar las regularidades estadísticas observadas, ya sea a las situaciones que de forma repetida se asocian a la ocurrencia del fenómeno, ya sea al estado de persona separada ${ }^{17}$. Y para ello, en el caso del divorcio, procesan información referida a diversas dimensiones significativas de la vida de las personas para la ocurrencia del fenómeno: el origen familiar, en la medida que, desde la más temprana socialización, moldea el sistema de valores de cada uno; las características de las uniones, que nos informan del significado atribuido al hecho de establecer un lazo con otra persona, y las características individuales, en la medida que definen el grado de autonomía individual dentro de la sociedad y de la propia unión.

En la práctica, en esta labor, los demógrafos estamos muy condicionados por la información de la que disponemos. En España, hasta el momento, la mejor fuente para el estudio de los determinantes del divorcio la constituye la ESD de 1991, porque es una fuente privilegiada para el estudio del fenómeno desde

15. La FFS se llevó a cabo entre octubre de 1988 y marzo de 1998 en 21 países europeos, Canadá, Nueva Zelanda y Estados Unidos. En abril y mayo de 2006, el Centro de Investigaciones Sociológicas llevó a cabo la encuesta Fecundidad y valores en la España del siglo XXI, aprovechando la tradición de captación de información retrospectiva iniciado con la FFS.

16. Son causas legales las siguientes: abandono injustificado de hogar, infidelidad conyugal, proceso penal (condena superior a seis años de prisión), conducta injuriosa o vejatoria o cualquier otra violación grave o reiterada de los deberes conyugales, maltratos, alcoholismo, toxicomanía, etc. En la primera versión del Boletín de sentencia de nulidad, separación o divorcio, vigente desde 1995 hasta diciembre de 1998, se incluían seis causas legales de nulidad, nueve de separación y seis de divorcio.

17. Los demógrafos, desde la primera tabla de mortalidad construida para la ciudad de Londres por John Graunt en 1666, están empeñados en dar con las regularidades estadísticas. 
una perspectiva biográfica. Además de las variables matrimoniales, puede utilizarse la información biográfica sobre la presencia de hijos menores de edad en la pareja, el nivel de educación de la mujer, su situación de ocupación, su residencia y las características sociodemográficas de sus padres. La encuesta permite seguir, año a año, la situación y la evolución de esas características, en el caso de que éstas cambien con el tiempo. Alguna de las variables consideradas como determinantes de la ruptura de las uniones cambian a lo largo de la biografía de la unión de las mujeres (como la presencia de hijos, la situación de ocupación, etc.), mientras que otras no lo hacen (como el municipio de residencia a los quince años, la edad en la que se inició la unión, la diferencia de edad entre los cónyuges, etc.). De esta manera, se pueden calcular las probabilidades de separación de las mujeres y de los hombres que viven en unión a cada momento (riesgo anual) según las características consideradas cada una por separado o en conjunto.

La ESD plantea algunas limitaciones, tanto en relación con el fenómeno en estudio como en relación con la metodología utilizada. En primer lugar, las historias matrimoniales son incompletas, pues las preguntas relacionadas con las uniones no permiten conocer la fecha de matrimonio, sino sólo la fecha de inicio de la unión. La consecuencia es que, por una parte, no podemos estudiar las rupturas de matrimonios en relación con la fecha del matrimonio mismo, y, por otra parte, no podemos estudiar los efectos de la cohabitación prematrimonial sobre las rupturas de matrimonios. En segundo lugar, la fecha de los acontecimientos normalmente aparece a escala anual y esto constituye un factor de imprecisión en los cálculos de la tasa de riesgo, sobre todo en lo que se refiere a determinar el orden de sucesión de los eventos y las relaciones entre causa y efecto que podrían establecerse entre ellos.

También la FFS contiene una amplia información retrospectiva sobre historia de uniones, biografía de la maternidad, historia contraceptiva, biografía de la educación y biografía laboral (sólo los períodos de ocupación de tres meses o más), organizada en episodios con fecha inicial y final —mes y añoy con una importante batería de variables que informan sobre cada episodio. Para el estudio de cualquier evento o proceso dependiente, toda esta información retrospectiva puede organizarse de forma que se conozca con gran nivel de detalle en qué situación laboral, matrimonial, educativa, reproductiva, etc. estaba el individuo en el momento de producirse el evento y cuáles son las circunstancias o las dimensiones personales y contextuales más importantes que caracterizan a dichas situaciones. En este sentido, se trata de una fuente interesante para analizar los determinantes del divorcio, pues se pueden utilizar la biografía de la educación y la biografía laboral como información que cambia con el tiempo.

La FFS también presenta una serie de limitaciones que dificultan el estudio de los determinantes del divorcio y que conviene mencionar aquí. En primer lugar, cabe subrayar que la encuesta sólo recoge las características de la pareja que tienen los individuos en el momento de realizar la entrevista, por lo que resulta imposible conocer característica alguna de los hombres y las mujeres 
que formaron una unión con los individuos entrevistados que posteriormente se rompió. En segundo lugar, cabe subrayar que, por razones de diseño del cuestionario, sólo es posible conocer el estado civil previo a la formación de la unión de los hombres y las mujeres que iniciaron una vida en común con los individuos encuestados sin casarse. Finalmente, es imprescindible citar algunas limitaciones que afectan a la construcción de otras variables de cierto poder explicativo. Citemos, por ejemplo, la ausencia de información relativa al tipo de contrato de trabajo y la imposibilidad de distinguir entre desempleo voluntario e involuntario, limitaciones que restringen sustancialmente la construcción de variables sociolaborales, y, finalmente, la falta de información sobre la región de residencia, condición que imposibilita apreciar la influencia de la diversidad política, socioeconómica y cultural de la geografía española ${ }^{18}$.

\subsection{Consecuencias de las rupturas de las uniones}

El estudio de las consecuencias inmediatas de la ruptura puede ser abordado desde la ESD, y los censos de población, en relación con las formas de convivencia que son un resultado de la ruptura, por ejemplo: ¿̨con quién viven los hijos? También es posible conocer las trayectorias posteriores a la ruptura en un horizonte más o menos lejano, a partir de las biografías de las uniones contenidas en las encuestas mencionadas (ESD y FFS) ${ }^{19}$ y a partir de datos publicados en el Movimiento natural de la población sobre la dinámica nupcial después del divorcio (en concreto, a partir del estado civil de los cónyuges en segundas nupcias o nupcias de orden superior).

\section{7. ¿Qué sabemos sobre el divorcio en España?}

Un estudio reciente sobre la evolución del divorcio en España desde 1981 hasta la actualidad ofrece la evolución de los flujos de divorcios y de los estocs de personas divorciadas (Solsona y Simó, 2007). En él se constata que, desde la aprobación de la ley de 1981, las cifras se han incrementado de forma paulatina, de manera que han pasado de 17.879 demandas de separación en el año 1982 a 73.567 en el año 2002. En todo el período 1982-2002, se totalizan más de un millón y medio de demandas de separación, divorcio y nulidad, lo cual, en términos de población, si no tuviéramos en cuenta la duplicidad en el proceso de ruptura (divorcio después de separación), significaría más de tres millones de personas implicadas. Al desagregar los datos, tenemos que, en este período 1982-2002, se han iniciado 890.375 procesos legales de separación y 592.111 procesos de divorcio, de los cuales, según los datos de 1996-2002, un $30 \%$ se habrían iniciado sin separación legal previa. Es decir, en conjunto,

18. La encuesta Fecundidad y valores en la España del siglo XXI, recientemente publicada por el Centro de Investigaciones Sociológicas, supera algunos de estos obstáculos.

19. Con la encuesta Fecundidad y valores en la España del siglo XXI es posible estudiar las trayectorias posteriores a la ruptura en el caso de las mujeres. 
más de dos millones de excónyuges han acudido a los tribunales por la ruptura de una unión matrimonial.

En cuanto a los estocs, las cifras son las siguientes: en 1981, la población cuyo estado civil era divorciada o separada no alcanzaba el cuarto de millón (241.131 personas); diez años más tarde, se acercaba al medio millón (455.875 personas), y a principios del tercer milenio, la cifra superaba generosamente el millón de personas (1.161.090 personas). Estas cifras impresionan, a pesar de que, en 2001, para el conjunto de la población mayor de quince años, estamos hablando del $2,85 \%$ de los hombres que viven en viviendas familiares y del 3,82\% de las mujeres, porque la cuestión es que la población divorciada y separada se concentra en unos grupos de edad específicos, por razones históricas obvias relativas a los cambios generacionales en el comportamiento familiar. Por ejemplo, para las mujeres de 45 años, en 2001, el porcentaje de separadas y divorciadas alcanzaba un 8,4\% (en 1991 era de un $3 \%$ y en 1981, de un 1,4\%). En el caso de los hombres de 45 años, estas cifras en 1981, 1991 y 2001 fueron, respectivamente: un 0,90\%, un 2,2\% y un $5,96 \%$.

Respecto a los factores determinantes, cuando iniciamos esta línea de investigación, planteamos una serie de hipótesis que el tiempo nos ha ayudado a confirmar o rechazar. La principal hipótesis de partida era que, a diferencia de lo que se señalaba en otros contextos donde el divorcio adquiere una intensidad mucho mayor y cuenta con una historia más larga, en España se trataría de un fenómeno social selectivo y menos normalizado como elemento estructural de la concepción de las relaciones de pareja. Desde la aprobación de la Ley del divorcio en 1981, el fenómeno ha crecido y se ha normalizado en España. Por otra parte, si la literatura reciente enfatizaba como factores determinantes del divorcio los factores individuales (como, por ejemplo, una edad temprana de acceso al matrimonio), en el caso de España consideramos que algunos factores de índole contextual (como el efecto de la generación, de la introducción de la reciente legislación sobre el divorcio, como las características de la familia de origen, como la región de pertenencia, etc.) desempeñarían un rol cuanto menos igual o de mayor relevancia en la determinación de las rupturas. Por último, dado que, en España, 1) la política social tiene como telón de fondo a la familia más que al individuo; 2) que, principalmente, los derechos sociales se derivan de la participación en el mercado de trabajo, y 3) que no existen medidas específicas para paliar los efectos que puedan generarse a nivel individual cuando acontece un divorcio, la independencia económica emergió como un factor determinante clave del divorcio.

En síntesis, en base al análisis de los datos de las fuentes presentadas más arriba, constatamos que la importancia de los factores determinantes del divorcio ha cambiado en las cohortes de unión más recientes, de manera que las variables de origen familiar condicionan en mayor medida a las promociones más antiguas, y las variables individuales (nivel educativo o relación de actividad), a las más jóvenes. 


\section{La experiencia europea en el registro estadístico del divorcio}

Al iniciar la redacción de este artículo, pensábamos que encontraríamos fuentes de inspiración en el proceder de nuestros países vecinos en el tema que nos atañe, pero lo cierto es que, en Francia e Inglaterra, sólo por tomar dos ejemplos de países donde las transformaciones demográficas de la familia empezaron a darse anteriormente, la situación no es tan diferente a la nuestra. En ambos países, el registro estadístico de la ruptura de las uniones presenta las mismas características que en el caso español. Es decir, únicamente quedan inscritas en el Registro Civil correspondiente las rupturas de uniones matrimoniales que implican un proceso legal: el État Civil, en el caso francés, y el Registrar Office, en el Reino Unido.

En Francia, con mucha más antigüedad que España, la recogida de información sobre las rupturas matrimoniales sigue criterios muy parecidos a los actuales de nuestro país, y el registro de divorcios en las estadísticas del estado civil obedece a un celo legislativo específico que obliga a registrar todo evento vital, incluido el matrimonio, y todo hecho que modifique el estado civil de las personas. Igual que en nuestro país, el divorcio consta inscrito al margen del acta matrimonial correspondiente. De 1970 a 1978, se confeccionaba una estadística especial sobre el divorcio que conllevaba una colecta importante de informaciones sobre las características sociodemográficas de los esposos y sobre aspectos judiciales del proceso. Desde 1980, la estadística de divorcios es competencia únicamente del Ministerio de Justicia.

La legislación reciente sobre las parejas de hecho cristalizó en Francia en el Pacto Civil de Solidaridad ${ }^{20}$ a finales de 1999, y el registro de estas uniones, como en España, queda fuera de las competencias del Registro Civil (INSEE, Résultats, Société, no 18, agosto de 2003).

En Inglaterra y Gales, también son los juzgados (las Cortes de Justicia) los que cumplimentan los boletines estadísticos de divorcio. Éstos incluyen la siguiente información: características sociodemográficas de los cónyuges, fecha de inicio y duración de las uniones, número de hijos dependientes y otros datos relativos al proceso, como la fecha de sentencia, inicio y duración del proceso, etc. Los juzgados son los encargados de remitir estos boletines cumplimentados a la Oficina Nacional de Estadísticas (National Statistics, 2001, Marriage, divorce and adoption Statistics. Review of the Registrar General on Marriages, divorces and adoptions in England and Walles, 1999, Series FM2, no 27).

Para conocer los estocs, igual que en el caso español, en la mayoría de los países europeos hay que recurrir a datos censales, mientras que para la observación de los determinantes del divorcio, y también para el estudio de las consecuencias que éste puede tener para los excónyuges y sus descendientes, algunos países, como Francia ${ }^{21}$, Gran Bretaña, los Países Bajos y Alemania, han avanzado mucho en la confección de encuestas específicas.

20. Este pacto es popularmente conocido como el PACS.

21. 2000-2001. Biographies et Entourage, 1996-1997. Étude sur le devenir des enfants naturels, 1994. Les situations familiales et l'emploi. 
Para profundizar en el estudio del fenómeno de las rupturas de unión, se ha constituido una red europea que se reúne anualmente. Su objetivo es promover el intercambio entre investigadores que realizan trabajos empíricos sobre diversos aspectos sociológicos de la ruptura de unión y profundizar en la perspectiva comparada. Dicha red pretende también organizar la publicación de las investigaciones que se realizan sobre este tema. Recientemente, esta red europea sobre divorcio ha celebrado su tercera conferencia en la ciudad alemana de Colonia ${ }^{22}$. Nos basamos en esta experiencia para resumir las últimas aportaciones europeas en esta cuestión y para presentar las encuestas existentes.

La Encuesta de fecundidad y familia (FFS) ha servido a diversos investigadores como fuente biográfica de datos para el estudio de las rupturas de unión en los siguientes ámbitos: el impacto de las variables macroeconómicas en Holanda, los factores del divorcio en Bélgica y, desde una perspectiva comparada incluyendo varios países europeos, el nivel educativo de las mujeres como determinante o la transmisión intergeneracional del divorcio.

Otras iniciativas en este terreno cuentan con fuentes de datos longitudinales de más complejidad. Nos referimos a la experiencia británica en la elaboración de encuestas de seguimiento longitudinal de cohortes específicas a lo largo del tiempo ${ }^{23}$. En el tema del divorcio, estas experiencias de recolección de datos han permitido realizar, a partir de la ola de 1970 y con el apoyo de una encuesta nacional especialmente diseñada para el estudio del desarrollo infantil ${ }^{24}$, una investigación sobre las consecuencias que ha tenido el divorcio de los padres en el bienestar de los hijos a corto y a largo plazo. También en Alemania, recientemente, se ha diseñado una encuesta específica para el estudio de las consecuencias del divorcio sobre la base de 1.519 personas divorciadas residentes en Alemania Oriental y Occidental, entrevistadas con un cuestionario retrospectivo durante los años 2000 y 2001. Además, en el caso de Alemania, el análisis de los factores de la ruptura de unión se centra en los sesgos de género relacionados con la iniciativa del proceso, explorando quién da el primer paso.

Quizás sea en el ámbito de las consecuencias donde, utilizando distintas metodologías diseñadas ad hoc, los temas tratados fueron más novedosos (redes familiares, consecuencias para la salud de los adultos, el síndrome de alineación parental, las transferencias intergeneracionales, etc.), sin olvidar el pionero estudio de las rupturas de uniones homosexuales en Noruega, que incluye un sugerente análisis de las diferencias entre las uniones de gais y lesbianas.

Por último, cabe apuntar que los estudios comparativos entre diferentes países europeos tuvieron también un espacio importante en esta tercera conferencia de Colonia, si bien el éxito de estos análisis está sujeto a la utilización

22. http://www.uni-koeln.de/wiso-fak/fisoz/conference/program/main.html.

23. Se trata de las diferentes olas de la British Cohort Study (1970, 1975, 1980, 1986, 1996 y 2000) realizadas por el Centre for Longitudinal Studies (http://www.cls.ioe.ac.uk/).

24. La National Child Development Survey (NCDS), basada en el seguimiento de 17.000 niños nacidos durante una semana de marzo del año 1958. 
de instrumentos de recogida de información homogéneos. En este sentido, nos congratulamos de la nueva iniciativa coordinada por el PAU de Ginebra (Unidad de Actividades de Población de Naciones Unidas), que, en cooperación con muchas otras instituciones ${ }^{25}$, ha puesto en marcha una nueva encuesta. Su principal objetivo es proporcionar información muy diversa en el tema de las rupturas de unión y en otros temas que explican el contexto social en el que los individuos toman sus decisiones. Se trata del Programa Generaciones y Género (GGS, de las siglas en inglés Generations and Gender Survey), en la que participan más de veinte países de toda Europa, Estados Unidos, Canadá y Nueva Zelanda.

El objetivo principal es estudiar los factores que explican la formación de la familia (matrimonio), la fecundidad y las relaciones entre generaciones, considerando tanto los factores individuales, como el entorno familiar y social y el contextual. La encuesta biográfica incluye historias de nacimientos, vida en pareja (cohabitaciones y matrimonios), educación, empleo y migraciones. Otros módulos versan sobre emancipación, contracepción, valores y actividad en el momento de la encuesta. Los datos biográficos permitirán realizar un estudio retrospectivo, pero, al mismo tiempo, se concibe como un estudio prospectivo (encuesta de panel), pues se van a entrevistar a las mismas personas tres veces en nueve años. El tamaño promedio de la muestra representativa a nivel nacional por país es de unos 10.000 individuos (hombres y mujeres de un amplio grupo de edades, de 17-79 años, para permitir el análisis de género y de generación).

Aunque cada país decide qué módulos se deben incluir y cuantas encuestas tienen que definir el panel, de entrada parece que la comparabilidad se garantizará entre los siguientes países: Francia, Rusia, Bulgaria, Alemania, Italia, Hungría (que está realizando ya la segunda vuelta), Austria y Polonia. Quién haya leído con atención este artículo hasta aquí, a estas alturas probablemente estará tan convencido como los autores que en esta lista de países debería figurar también España.

\section{Conclusiones e ideas para avanzar}

Es evidente que observar una ruptura de una unión es más complicado que el registro de un nacimiento o una defunción, eventos que, por otra parte, tienen un referente temporal bien delimitado. Sin embargo, la precariedad estadística sobre el divorcio en España se explica por la falta de mecanismos de observación continua de los cambios demográficos de comportamiento, que nos permitan conocer mejor sus tendencias y generar un conocimiento útil, no sólo para satisfacer las inquietudes intelectuales de los investigadores socia-

25. Algunas de estas instituciones son las siguientes: Max Plank Institute de Rostock (Alemania), Centre for Analysis of Social Exclusion (Reino Unido), Departamento de la Universidad de Roma "La Sapienza» (Italia), Hungarian Statistical Office (Hungria), INED (Francia), Netherlands Interdisciplinary Demographic Institute (Holanda) y Statistics Canada (Canadá). 
les, sino también para un buen diseño de políticas públicas en este ámbito. Según las recomendaciones de Naciones Unidas, estos mecanismos de observación continua deberían formar parte del Registro Civil, pero en España, en virtud del acuerdo firmado en 1995 entre el INE y el CGPJ, la elaboración de datos estadísticos de divorcios y separaciones, mediante la implementación de los boletines estadísticos mencionados, queda casi exclusivamente en manos de la administración de la justicia, si bien el Instituto Nacional de Estadística se encarga de recopilarlos, tabularlos y publicitarlos, como hace con las estadísticas del Registro Civil de nacimientos, defunciones y matrimonios.

En el caso español, únicamente la existencia de encuestas biográficas (de 1991 y 1995) nos ha permitido explorar las características demográficas de las rupturas de las uniones en España de una forma completa, ofreciendo estimaciones de su acaecimiento y las características de sus protagonistas para diferentes generaciones y promociones de matrimonio (tabla 2). Como quiera que estamos tratando de un fenómeno que, con el tiempo, deviene más intenso y más complejo, y que las estimaciones de las que disponemos son ya demasiado lejanas, necesitamos medir y conocer mejor el proceso de transformación de las uniones en tiempos recientes. Para ello, proponemos, como medida inmediata, la elaboración de una encuesta retrospectiva que permita seguir la supervivencia de las promociones de unión actuales, y como sistema más permanente, una encuesta en panel que vaya integrando los cambios en el tiempo de las promociones de unión existentes, y que, de forma continua, vaya introduciendo las nuevas promociones de unión.

En este momento en el que se está fraguando un importante proyecto europeo denominado Generations and Gender Survey, que incluye un módulo sobre la historia de las uniones, quizás la elaboración de una encuesta específica para España no sea la mejor alternativa. Más bien deberíamos proponer la vinculación de España en este proyecto de demografía comparada.

Respecto a la observación continua de las rupturas de las uniones, si bien, al iniciar la redacción de este artículo, pensábamos que encontraríamos fuentes de inspiración en el proceder de nuestros países vecinos en el tema que nos atañe, lo cierto es que, en Francia e Inglaterra, la situación no es tan diferente a la nuestra. En consecuencia, dadas las semejanzas en el contexto europeo y la necesidad también compartida de adaptar las estadísticas a los cambios, pensamos que es un momento ideal para reflexionar conjuntamente sobre la orientación a seguir en la mejora de la observación del fenómeno de las rupturas de unión. Ello, según nuestra opinión, tendría que ver con una unificación y una simplificación de las fuentes existentes, en un nuevo registro civil. Un nuevo registro civil que incluyera todo tipo de uniones, no únicamente las matrimoniales.

En este sentido, el reconocimiento de derechos que están solicitando las parejas de hecho y la progresiva equiparación con las uniones matrimoniales, junto con el reconocimiento del derecho al matrimonio de las uniones homosexuales, apuntan también hacia una unificación y una simplificación del registro de la formación y de la ruptura. Ello también debería suponer, nos atrevemos 


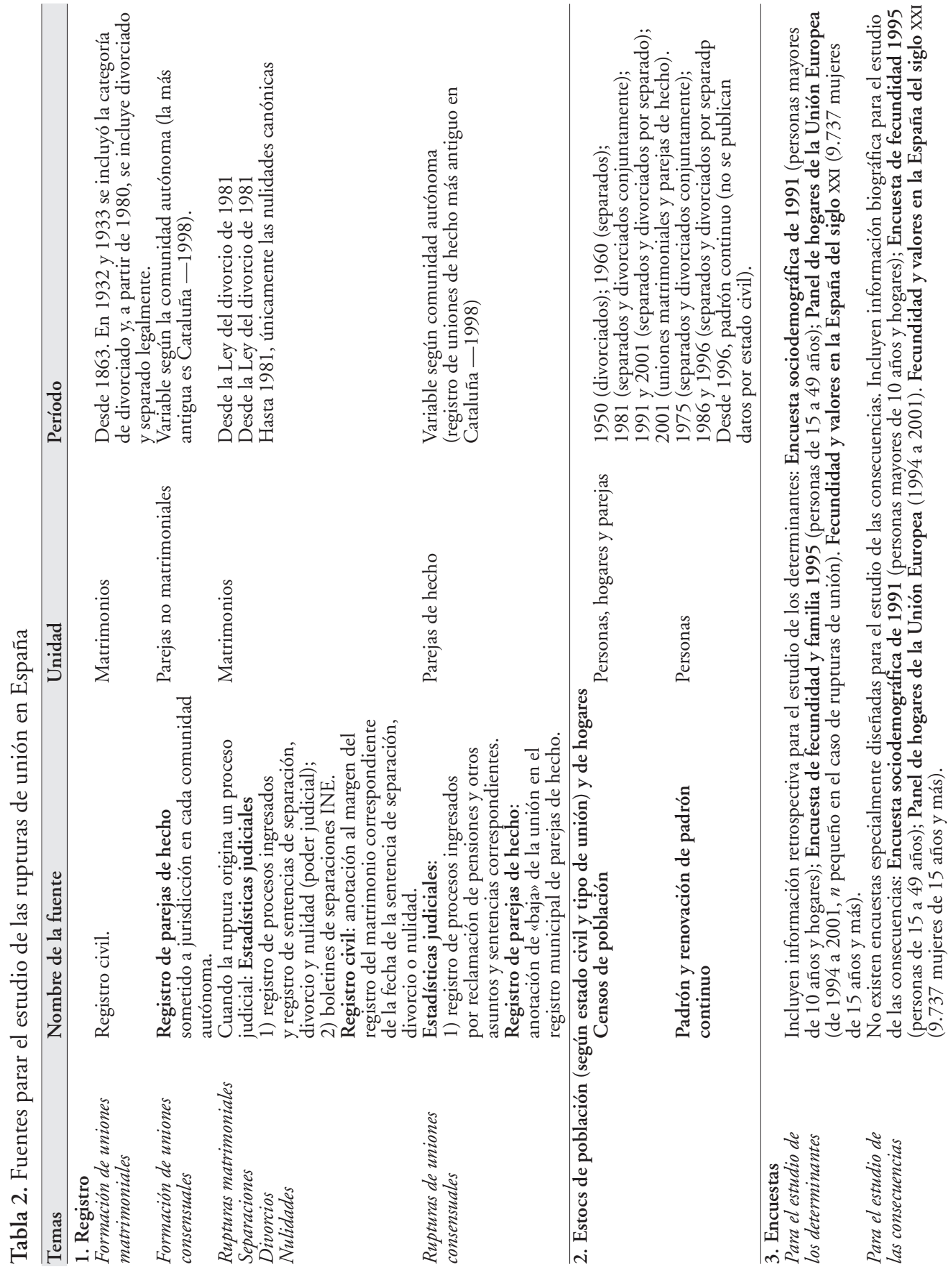


a sugerir, la adopción de medidas que permitieran integrar definitivamente la inscripción de las rupturas de todo tipo de unión formalizada en el Registro Civil, como principal vía para reconocer los derechos y los deberes correspondientes a toda forma de unión y, por tanto, garantizar unas condiciones claras para todos en caso de ruptura.

Finalmente, para el estudio de las consecuencias de las rupturas, proponemos una encuesta en panel (que incluya preguntas retrospectivas) que siga paulatinamente a las personas separadas y divorciadas y que combine las diferentes perspectivas socioeconómica, laboral (ocupacional), relacional, etc., así como que también tenga en cuenta los diferentes agentes afectados (hombre, mujer, hijos menores, hijos emancipados, etc.).

\section{Referencias bibliográficas}

Delgado, Margarita y CASTRO MARTín, Teresa (1999). Encuesta de fecundidad y familia 1995. Madrid: Centro de Investigaciones Sociológicas.

Houle, René (2004). The effects of socio-cultural and labor market conditions on marital separation during the early democratic period in Spain. Working Paper WP 2004023. Max-Planck Oinstitute for Demographic Research.

Houle, René; Simó, Carles; Solsona, Montserrat y TREviÑo, Rocío (1999). «Análisis biográfico del divorcio en España». Revista Española de Investigaciones Sociológicas, no 88 (octubre-diciembre), 11-35.

NACIONES UNIDAS (1998). Grupo de expertos sobre el proyecto de principios y recomendaciones para un sistema de estadística vitales. Consejo Económico y Social. Comisión de Estadística.

SimÓ, Carles y Solsona, Montserrat (2003). «Importancia de la participación laboral como determinante del divorcio en España». Papeles de Geografia, 37, 245-259. Universidad de Murcia.

Simó, Carles; Solsona Montserrat; Houle, René y Treviño, Rocío (2000). «Els determinants sociodemogràfics de les ruptures de les unions a Catalunya». Revista Catalana de Sociología, $\mathrm{n}^{\circ}$ 12. Monogràfic «Families: espais, ruptures i polítiques», $87-110$.

Solsona, Montserrat (2002). «Família». En: Giner, Salvador (dir.). Enquesta de la Regió de Barcelona 2000. Institut d'Estudis Regionals i Metropolitans, cap. VI, 139-162. [http://campus.uab.es/iermb/enquesta 2000/indexerb.htm]

Solsona, Montserrat, Simó, Carles y Houle, René (1999) «Separation and divorce in Spain in the context of the European Community». South European Society and Politics, vol. 4, no 2 (otoño), 195-222.

- (2000). «Separation and divorce in Spain». En: GonZÁlez, M.J.; JuRAdo, T. y NALDINI, M. (eds.). Gender inequalities in Southern Europe: Women, work and welfare in the 1990s. Londres: Frank Cass, 195-222.

Treviño, Rocío, Solsona, Montserrat; Simó, Carles y Houle, René (2000). «Los determinantes sociodemográficos y familiares de las rupturas de uniones en España: La normalización del fenómeno». Boletín de la Asociación de Demografia Histórica, XVIII, I, 101-235. 